\title{
Robotic Surgery for Rectal Cancer Provides Advantageous Outcomes Over Laparoscopic Approach
}

\author{
Results From a Large Retrospective Cohort
}

\author{
Jacopo Crippa, MD, * Fabian Grass, MD, ${ }^{*}$ Eric J. Dozois, MD, ${ }^{*}$ Kellie L. Mathis, MD, ${ }^{*}$ Amit Merchea, MD,$\dagger$ \\ Dorin T. Colibaseanu, MD, $\dagger$ Scott R. Kelley, MD, ${ }^{*}$ and David W. Larson, MD, MBA*凶
}

\begin{abstract}
Objective: To compare short term outcomes of patients undergoing laparoscopic or robotic rectal cancer surgery.

Background: Significant benefits of robotic rectal cancer surgery over laparoscopy have yet to be demonstrated. Operative time and direct institutional cost seem in favor of the laparoscopic approach.

Methods: We performed a retrospective review of consecutive patients operated on for rectal cancer with a mini-invasive approach at Mayo Clinic from 2005 to 2018 . The primary aim of this study was to investigate the difference in postoperative morbidity between the laparoscopic and robotic approach. Multivariable models for odds to complications and prolonged $(\geq 6$ days) length of stay were built.

Results: A total of 600 patients were included in the analysis. The number of patients undergoing robotic surgery was $317(52.8 \%)$. The 2 groups were similar in respect to age, sex, and body mass index. Laparoscopic surgery was correlated to shorter operative time (214 vs 324 minutes; $P<0.001$ ). Patients undergoing robotic surgery had a lower overall complications rate $(37.2 \%$ vs $51.2 \% ; P<0.001)$. Robotic surgery was found to be the most protective factor [odds ratio (OR) $0.485 ; P=0.006$ ] for odds to complications. The event of a complication (OR 9.33; $P<0.001$ ) and conversion to open surgery (OR $3.095 ; P=0.002)$ were identified as risk factors for prolonged length of stay whereas robotic surgery (OR $0.62 ; P=0.027$ ) was the only independent protective factor.
\end{abstract}

Conclusions: Robotic rectal cancer surgery is strongly associated with better short-term outcomes over laparoscopic surgery.

Keywords: complications, laparoscopic surgery, rectal cancer, rectal surgery, robotic surgery, surgical outcomes

(Ann Surg 2021;274:e1218-e1222)

$\mathrm{T}$ he multidisciplinary treatment for rectal cancer is a field of continuous innovation. Despite recent progress in perioperative management, radical resection still represents the only chance for definitive cure. ${ }^{1}$ Achieving optimal surgical and oncological quality is nonetheless challenging. Although a total mesorectal excision (TME) yielding negative circumferential margins is key to optimal local control, surgery can result in devastating consequences, ${ }^{2}$ affecting both short- and long-term functional outcomes. ${ }^{3-5}$ Quality of life and minimization of risk and adverse events is at the core of patient-centered decision making for the treatment of rectal cancer. ${ }^{6}$

From the *Division of Colon \& Rectal Surgery, Mayo Clinic, Rochester, Minnesota; and $\dagger$ Division of Colon \& Rectal Surgery, Mayo Clinic, Jacksonville, Florida.

\Larson.David2@mayo.edu.

This research did not receive any specific grant from funding agencies in the public, commercial, or not-for-profit sectors.

The authors report no conflicts of interest.

Copyright (C) 2020 Wolters Kluwer Health, Inc. All rights reserved.

ISSN: 0003-4932/20/27406-e1218

DOI: $10.1097 /$ SLA.0000000000003805
New surgical technologies aim to overcome the challenge of the narrow pelvic space and technically demanding dissection typical of pelvic cancer surgery. Laparoscopic technique has been described as noninferior to open rectal cancer surgery for short-term outcomes within European trials, ${ }^{7}$ and these findings were later supported by similar oncological outcomes in an American based trial. ${ }^{8}$ However, significant benefits of robotic rectal cancer surgery over laparoscopy have yet to be demonstrated. In fact, previous trials have failed to reveal a clear advantage of the robotic technique; moreover, operative time and direct institutional costs seem to support a laparoscopic approach. ${ }^{9}$ Nevertheless, quality of data is a current limitation with the majority deriving from small cohorts. ${ }^{10}$ Recent results from a large randomized controlled trial (RCT) were powered to address conversion rather than postoperative outcomes and may have been influenced by surgeons' learning curve. ${ }^{11}$ Therefore, further reliable data is needed to minimize nonsignificant results that could lead to misinterpretations. ${ }^{12}$

The aim of the present study is to compare 30-day outcomes of a large cohort of patients undergoing laparoscopic or robotic rectal cancer surgery at a tertiary cancer center.

\section{METHODS}

The Institutional Review Board approved this retrospective analysis of a prospectively maintained database of consecutive patients operated on for rectal cancer from February 2005 to April 2018 at Mayo Clinic, Rochester, Minnesota and Mayo Clinic, Jacksonville, Florida. Inclusion criteria were elective curative-intent surgery for rectal adenocarcinoma, minimally-invasive approach, and age older than 18 years. Exclusion criteria were stage IV or recurrent disease, lack of research authorization, and emergency surgery. All operations were performed by 10 institutional boardcertified colorectal surgeons. Pertinent variables of interest were demographics, tumor characteristics, specifics on oncological treatment, robotic or laparoscopic approach, and postoperative outcomes.

The primary aim of this study was to investigate the difference in postoperative morbidity between the laparoscopic and robotic approach.

Outcomes of interest were length of stay (LOS), 30-day readmission rate, need for transfusion, postoperative ileus, wound infection, anastomotic leak, and overall complication rate. Anastomotic leak was defined according to the classification proposed by the International Study Group of Rectal Cancer and assessed for patients undergoing restorative surgery. ${ }^{13}$ Postoperative ileus was defined as postoperative prolonged ( $>3$ days) nil per os or need of naso-gastric tube insertion.

Overall complications included both surgical and nonsurgical. Surgical complications included ileus, anastomotic leak, postoperative need for blood transfusion, and wound infection. Nonsurgical complications included cardiovascular and pulmonary complications, urinary tract infections and acute kidney injury. Postoperative 
anemia was defined as hemoglobin level $<13 \mathrm{~g} / \mathrm{dL}$ for men and $<12 \mathrm{~g} / \mathrm{dl}$ for women. ${ }^{14}$ Blood transfusion is administered when patients developed symptoms related to anemia or when hemoglobin levels reached $<7 \mathrm{~g} / \mathrm{dL}(<10 \mathrm{~g} / \mathrm{dL}$ in patients with a history of cardiac disease). All complications were assessed in-hospital and until 30 days postoperatively.

Further-analysis of patients requiring prolonged hospitalization (LOS $\geq 6$ days) was performed to investigate associated risk factors. A cut-off of 6 days was chosen as it represents the 75th percentile of LOS in our cohort.

Methodology for staging and preoperative evaluation is described in a previous paper from our institution, ${ }^{15}$ and description of robotic ${ }^{15}$ and laparoscopic ${ }^{16}$ techniques. A multimodal colorectal enhanced recovery program (ERP) was established in our institution as standard perioperative care protocol starting from $2010 .{ }^{17}$ The first robotic procedure was performed in 2007 whereas a structured colorectal robotic program began in 2010. Four surgeons switched to robotics for their entire minimally-invasive practice because the implementation of the robotic platform, 3 surgeons were hired during the study period and performed exclusively robotic whereas others did not change from laparoscopy to robotics $(n=3)$.

\section{Statistical Analysis}

Continuous variables were described as mean (standard deviation) or median (interquartile range-range) as appropriate; categorical variables as frequencies and percentage. Significant differences between the 2 groups were tested by $\chi^{2}$ or Fischer exact test for categorical variables and Student $t$ test for continuous variables.

Univariate analysis for odds to any complication and LOS $\geq 6$ days was performed by logistic regression for every confounder from our database; a multivariable model was built considering significant $(P<0.05)$ variables from the univariate regression; results are shown as odds ratio (OR) [95\% confidence interval]. All tests were 2 -sided, a $P$-value $<0.05$ was considered statistically significant. Analysis was performed using JMPPro (Version 13.0, SAS Institute, Cary, $\mathrm{NC})$.

\section{RESULTS}

Of the 600 included patients, 317 (52.8\%) underwent robotic surgery and 283 were $(47.2 \%)$ treated laparoscopically.

Table 1 reports demographics and specifics on tumor stage, oncological and surgical treatment. The 2 groups were similar in respect to age, sex, and body mass index. The robotic group comprised a larger percentage of stage III patients $(63.5 \%$ vs $50.2 \% ; P=0.001)$ and a higher rate of patients undergoing neoadjuvant therapy $(68.8 \%$ vs $44.9 \% ; P<0.001)$. ERP was the standard perioperative protocol for most patients of the robotic but not the laparoscopic group $(84.5 \%$ vs $18.4 \% ; P<0.001)$. Patients treated laparoscopically underwent restorative surgery more frequently $(78.8 \%$ vs $66.6 \% ; P<0.001)$ than those treated robotically, and had a shorter operative time (214.6 vs 324.1 minutes; $P<0.001$ ).

Table 2 reports postoperative outcomes. Patients undergoing robotic surgery had a lower overall complications rate $(37.2 \%$ vs $51.2 \% ; P<0.001)$ and lower transfusion requirements $(1.9 \%$ vs $7.8 \% ; P<0.001)$. The 2 groups had similar readmission, ileus, and anastomotic leak rate. Median LOS ( 3 vs 5 days; $P<0.001$ ), and prolonged LOS $(21.45 \%$ vs $43.11 \% ; P<0.001)$ were in favor of the robotic group.

\section{Logistic Regression Analysis}

Univariate analysis for odds to any complication revealed multiple items to be significant predictors. Male sex, obesity, type of procedure, age, operative time, surgical approach, and ERP were retained as significant in the multivariable model. Robotic surgery was found to be the most protective factor (OR $0.485 ; P=0.006)$ for odds to complications (Table 3 ).

Univariate analysis for odds to LOS $\geq 6$ days (Table 4) had 8 significant variables that were included in the multivariable model.

TABLE 1. Demographics and Treatment

\begin{tabular}{|c|c|c|c|c|}
\hline & Robotic (317) & Laparoscopic (283) & Total $(600)$ & $P$-value \\
\hline Age & & & & 0.795 \\
\hline Median (IQR) & $58(50-68)$ & $59(50-69)$ & $59(50-68.2)$ & \\
\hline Sex (male) & $210(66.25 \%)$ & $193(68.2 \%)$ & $403(67.2 \%)$ & 0.611 \\
\hline Mean (SD) & $27.6(5.3)$ & $27.6(5.0)$ & $27.6(5.2)$ & \\
\hline ERP & $268(84.5 \%)$ & $52(18.4 \%)$ & $320(53.3 \%)$ & $<0.001$ \\
\hline ASA & & & & 0.031 \\
\hline 4 & $1(0.3 \%)$ & $2(0.7 \%)$ & $3(0.5 \%)$ & \\
\hline Treated Stage & & & & $0.002 *$ \\
\hline I & $60(18.9 \%)$ & $86(30.4 \%)$ & $146(24 \%)$ & \\
\hline II & $58(18.3 \%)$ & $55(19.4 \%)$ & $113(18.8 \%)$ & \\
\hline III & $199(62.8 \%)$ & $142(50.2 \%)$ & $341(57.1 \%)$ & \\
\hline Neoadjuvant therapy & $218(68.8 \%)$ & $127(44.9 \%)$ & $345(57.5 \%)$ & $<0.001$ \\
\hline Mean (SD) & $324.1(108.4)$ & $214.6(71)$ & $272(107.5)$ & \\
\hline Conversion & $16(5.05 \%)$ & $39(13.8 \%)$ & $55(9.2 \%)$ & $<0.001$ \\
\hline Positive CRM & $1(0.3 \%)$ & $4(1.3 \%)$ & $5(0.83 \%)$ & 0.379 \\
\hline
\end{tabular}

APR indicates abdominoperineal resection; ASA, American Society of Anesthesiologists Classification; BMI, body mass index; CRM, circumferential radial margin; ERP, enhanced recovery pathway; IQR, interquartile range; LAR, low anterior resection; SD, standard deviation. 
TABLE 2. Postoperative Outcomes

\begin{tabular}{|c|c|c|c|c|}
\hline & Robotic (317) & Laparoscopic (283) & Total (600) & $P$-value \\
\hline LOS & & & & $<0.001$ \\
\hline Median (IQR) & $3(3-5)$ & $5(4-7)$ & $4(3-6)$ & \\
\hline LOS $\geq 6 \mathrm{~d}$ & $68(21.45 \%)$ & $122(43.11 \%)$ & $190(31.7 \%)$ & $<0.001$ \\
\hline Readmission & $43(13.6 \%)$ & $30(10.6 \%)$ & $73(12.2 \%)$ & 0.266 \\
\hline Overall complication & $118(37.2 \%)$ & $145(51.2 \%)$ & $263(43.8 \%)$ & $<0.001$ \\
\hline Anemia & $13(4.1 \%)$ & $28(9.9 \%)$ & $41(6.8 \%)$ & $<0.005$ \\
\hline Transfusion & $6(1.9 \%)$ & $22(7.8 \%)$ & $28(4.7 \%)$ & $<0.001$ \\
\hline Ileus & $50(15.8 \%)$ & $51(18.0 \%)$ & $101(16.8 \%)$ & 0.463 \\
\hline Leak $^{*}$ & $23(10.9 \%)$ & $14(8.9 \%)$ & $37(8.5 \%)$ & 0.238 \\
\hline Superficial wound infection & $20(6.3 \%)$ & $15(5.3 \%)$ & $35(5.83 \%)$ & 0.598 \\
\hline Cardiopulmonary complications & $16(5.05 \%)$ & $20(7.07 \%)$ & $36(6 \%)$ & 0.299 \\
\hline UTI & $5(1.6 \%)$ & $14(4.95 \%)$ & $19(3.2 \%)$ & 0.020 \\
\hline Acute kidney injury & $6(1.89 \%)$ & $8(2.83 \%)$ & $14(2.33 \%)$ & 0.449 \\
\hline Adjuvant therapy & $179(57.2 \%)$ & $149(52.65 \%)$ & $328(55.0 \%)$ & 0.266 \\
\hline Death $<30 \mathrm{~d}$ & $1(0.3 \%)$ & $1(0.3 \%)$ & $2(0.3 \%)$ & 0.936 \\
\hline
\end{tabular}

${ }^{*}$ Assessed for patients undergoing restorative surgery.

IQR indicates interquartile range; LOS, length of stay; UTI, urinary tract infection.

The event of a complication (OR 9.33; $P<0.001)$ and conversion to open surgery (OR $3.095 ; P=0.002$ ) were identified as risk factors for prolonged LOS whereas robotic surgery (OR $0.62 P=0.027)$ was the only independent protective factor.

\section{DISCUSSION}

The present investigation on short term outcomes of a large cohort of patients undergoing robotic and laparoscopic surgery for rectal cancer at a tertiary cancer center suggests that robotic surgery was advantageous over laparoscopic surgery in terms of postoperative morbidity and length of hospital stay. Patients undergoing robotic surgery were $51 \%$ and $38 \%$ less likely to experience a complication or a hospitalization $\geq 6$ days, respectively, compared to laparoscopic surgery.

Prete et al recently published a meta-analysis of 5 RCTs involving 671 patients on robotic surgery for rectal cancer compared to the laparoscopic approach. ${ }^{18}$ They found robotic surgery to be associated with a lower conversion rate and longer operative time, whereas overall short-term morbidity was equal between the 2 groups. Our study of 600 minimally invasive procedures found similar results for conversion rate and operative time. Furthermore, a strong association between robotic surgery and decreased postoperative morbidity was observed. The majority of the RCTs included in the meta-analysis reports on small sample size and may; therefore, lack in power. This reflects the difficulties of planning and performing a RCT on robotic surgery. Reasons for this could be high costs, lack of multicenter collaboration, and heterogeneous surgeons' experience. Large retrospective cohorts like ours, could be helpful to provide reliable data and overcome these limitations.

In 2016, Sun et al published a meta-analysis of 592 cases (324 robotic/268 laparoscopic), ${ }^{19}$ reporting a weak association between lower complication rate and robotic rectal surgery. In our series, robotic surgery was the most significant protective factor for the event of complication and the only protective factor against prolonged LOS.

To date, this is the largest published retrospective series of patients undergoing minimally invasive rectal cancer surgery comparing outcomes of a robotic versus laparoscopic approach. Numerous smaller case-series have been previously published to investigate the superiority of either technique, but results are frequently inconclusive. ${ }^{19}$ Nevertheless, multiple attempts at systematic reviews and meta-analyses have been made, and yet results remain uncertain. ${ }^{10}$ Of note, nowadays MIS accounts for the majority of the rectal cancer cases performed at our institution in the elective setting as only $20 \%$

TABLE 3. Multivariable Model for Odds to Any Complication

\begin{tabular}{|c|c|c|c|c|}
\hline Logistic Regression for Odds to Any Complication & Univariate OR (CI 95\%) & $P$-value & Multivariable OR (CI 95\%) & $P$-value \\
\hline Sex (male vs female) & $1.9(1.33-2.71)$ & $<0.001^{*}$ & $1.58(1.08-2.32)$ & $0.019^{*}$ \\
\hline BMI ( $\geq 30$ vs $\left.<30 \mathrm{~kg} / \mathrm{m}^{2}\right)$ & $1.75(1.21-2.53)$ & $0.002^{*}$ & $1.51(1.01-2.25)$ & $0.044^{*}$ \\
\hline Procedure (LAR vs APR) & $0.64(0.45-0.92)$ & $<0.015^{*}$ & $0.6(0.4-0.9)$ & $0.014^{*}$ \\
\hline Age & $1.03(1.02-1.04)$ & $<0.001^{*}$ & $1.02(1.001-1.04)$ & $0.001^{*}$ \\
\hline Operative time & $1.002(1.0001-1.003)$ & $0.029^{*}$ & $1.004(1.002-1.006)$ & $<0.001^{*}$ \\
\hline ERP (yes vs no) & $0.45(0.32-0.62)$ & $<0.001^{*}$ & $0.53(0.33-0.84)$ & $0.007^{*}$ \\
\hline \multicolumn{5}{|l|}{ ASA (reference I-II) } \\
\hline \multicolumn{5}{|l|}{ Treated stage (reference I) } \\
\hline II & $1.04(0.63-1.71)$ & 0.228 & & \\
\hline III & $1.12(0.75-1.66)$ & 0.139 & & \\
\hline Neoadjuvant therapy & $1.08(0.78-1.5)$ & 0.644 & & \\
\hline
\end{tabular}

APR indicates abdominoperineal resection; ASA, American Society of Anesthesiologists Classification; BMI, body mass index; CI, confidence Interval; ERP, enhanced recovery pathway; LAR, low anterior resection; OR, odds ratio.

*Statistically significant. 
TABLE 4. Multivariable Model for Odds to LOS $\geq 6 \mathrm{~d}$

\begin{tabular}{|c|c|c|c|c|}
\hline Logistic Regress for Odds to LOS $\geq 6$ & Univariate OR (CI 95\%) & $P$-value & Multivariable OR (CI 95\%) & $P$-value \\
\hline Sex (male vs female) & $0.53(0.36-0.78)$ & $0.001^{*}$ & $1.41(0.88-2.26)$ & 0.148 \\
\hline BMI $\left(\geq 30\right.$ vs $\left.<30 \mathrm{~kg} / \mathrm{m}^{2}\right)$ & $1.89(1.29-2.75)$ & $0.001^{*}$ & $1.41(0.89-1.08)$ & 0.146 \\
\hline Procedure (LAR vs APR) & $0.88(0.6-1.28)$ & 0.501 & - & - \\
\hline Age & $3.23(1.24-8.41)$ & $0.016^{*}$ & $0.99(0.98-1.01)$ & 0.717 \\
\hline Operative time & $1.001(0.999-1.002)$ & 0.341 & - & - \\
\hline ERP (yes vs no) & $0.31(0.22-0.45)$ & $<0.001^{*}$ & $0.62(0.36-1.08)$ & 0.092 \\
\hline Any complication & $10.77(7.1-16.35)$ & $<0.001^{*}$ & $9.33(5.97-14.57)$ & $<0.001^{*}$ \\
\hline \multicolumn{5}{|l|}{ Treated stage (reference I) } \\
\hline II & $1.093(0.64-1.87)$ & 0.746 & & \\
\hline III & $1.66(0.76-1.78)$ & 0.480 & & \\
\hline Neoadjuvant therapy & $0.93(0.66-1.32)$ & 0.690 & & \\
\hline
\end{tabular}

APR indicates abdominoperineal resection; ASA, American Society of Anesthesiologists Classification; BMI, body mass index; CI, confidence interval; ERP, enhanced recovery pathway; LAR, low anterior resection; OR, odds ratio.

${ }^{*}$ Statistically significant.

of our patients undergo open surgery. Our report from a cancer center with long-standing experience in MIS may contribute further evidence in this highly debated field.

Surgical innovations should follow a systematic pathway to validation. ${ }^{20}$ To date, the decision to choose a robotic approach for rectal cancer surgery is still controversial, often driven by surgeons' preference rather than evidence-based considerations. Safety and feasibility have been frequently reported but analysis of postoperative outcomes remains inconclusive. ${ }^{21,22}$ Analysis of costs often refers to short time frames and this could mislead one to assume that higher operative costs of robotics are not balanced by favorable outcomes. ${ }^{23}$ Although intraoperative outcomes are frequently in favor of robotic surgery, ${ }^{24,25}$ postoperative short term outcomes are reported to be similar between the 2 approaches.

Robotic surgery was associated with prolonged operative time in our series and this is consistent with previous evidence. ${ }^{19}$ In our multivariable model of complications, longer operative time was retained as a risk factor. Although robotic resection was associated with a longer operative time compared to laparoscopy, prolonged surgical time did barely impact the complication rate, given the independent protective effect of robotic surgery. Analysis of specific surgical complications revealed that both postoperative anemia and need for transfusion were lower in the robotic group. This may be a consequence of the precision of the technique through improved vision and tremorless motion. However, this would need confirmation by studies tailored to assess these specific outcomes. Median LOS was lower for the robotic group. This could arguably be a consequence of the higher proportion of ERP patients in the robotic cohort. In fact, due to the long study period with inherent differences in timing of patient enrollment due to surgical innovation and improvements in perioperative care, ERP was the standard perioperative protocol for most patients in the robotic but not for those in the laparoscopic group. Repeatedly, ERP has been strongly associated with improved short term outcomes such as shorter LOS and reduced complication rate. ${ }^{26-29}$ Interestingly, our multivariable logistic regression models for both complications and prolonged LOS including ERP as a confounder suggest robotic surgery to have an independent protective effect beyond enhanced recovery care.

Until now, robotic surgery has been associated mainly with a lower conversion rate, although results from the largest RCT available on robotic rectal surgery failed to prove its superiority over laparoscopy. ${ }^{30}$ Our findings of a strong association between the robotic approach to rectal cancer and fewer postoperative complications adds substantially to the current literature in the field.

Limitations of our study include but are not limited to its retrospective, nonrandomized nature. Despite analysis of a comprehensive list of potential confounders, the list is not exhaustive and further factors, notably subjective surgeons' preference for either approach, may have affected the results. Therefore, there is an inherent risk of selection bias and results must be interpreted accordingly. However, the large sample size, consecutive inclusion of all patients and the highly standardized surgical approach for either technique may limit the risk of systematic error related to sample selection. Furthermore, most surgeons adhered to an "all or nothing" practice change to the robotic approach, limiting thus the risk of true patient selection. Most laparoscopic cases were performed before the introduction of the robotic program. Advances in perioperative and oncological care in the highly specialized management of rectal cancer inevitably occurred over the long study period. This inevitably leads to inherent bias related to the long inclusion period. Thus, results need to be interpreted considering limitations related to the retrospective, nonrandomized study design.

In conclusion, the present study demonstrated improved short term outcomes of robotic surgery over laparoscopy in a large cohort of patients undergoing rectal resection for cancer and opens new perspective in the surgical approach to rectal cancer.

\section{REFERENCES}

1. Marijnen CA. Organ preservation in rectal cancer: have all questions been answered? Lancet Oncol. 2015;16:e13-e22.

2. Kim JY, Kim NK, Lee KY, et al. A comparative study of voiding and sexual function after total mesorectal excision with autonomic nerve preservation for rectal cancer: laparoscopic versus robotic surgery. Ann Surg Oncol. 2012;19:2485-2493.

3. Attaallah W, Ertekin C, Tinay I, et al. High rate of sexual dysfunction following surgery for rectal cancer. Ann Coloproctol. 2014;30:210-215.

4. Wiltink LM, Marijnen CA, Meershoek-Klein Kranenbarg E, et al. A comprehensive longitudinal overview of health-related quality of life and symptoms after treatment for rectal cancer in the TME trial. Acta Oncol. 2016;55:502-508.

5. Giandomenico F, Gavaruzzi T, Lotto L, et al. Quality of life after surgery for rectal cancer: a systematic review of comparisons with the general population. Expert Rev Gastroenterol Hepatol. 2015;9:1227-1242.

6. Mari GM, Crippa J, Cocozza E, et al. Low ligation of inferior mesenteric artery in laparoscopic anterior resection for rectal cancer reduces genitourinary dysfunction: results from a randomized controlled trial (HIGHLOW trial). Ann Surg. 2018;269:1018-1024. 
7. van der Pas MHGM, Haglind E, Cuesta MA, et al. Laparoscopic versus open surgery for rectal cancer (COLOR II): short-term outcomes of a randomised, phase 3 trial. Lancet Oncol. 2013;14:210-218.

8. Fleshman J, Branda ME, Sargent DJ, et al. Disease-free survival and local recurrence for laparoscopic resection compared with open resection of stage II to III rectal cancer: follow-up results of the ACOSOG Z6051 randomized controlled trial. Ann Surg. 2018;269:589-595.

9. Li X, Wang T, Yao L, et al. The safety and effectiveness of robot-assisted versus laparoscopic TME in patients with rectal cancer: a meta-analysis and systematic review. Medicine (Baltimore). 2017;96:e7585

10. Holmer C, Kreis ME. Systematic review of robotic low anterior resection for rectal cancer. Surg Endosc. 2018;32:569-581.

11. Corrigan N, Marshall H, Croft J, et al. Exploring and adjusting for potential learning effects in ROLARR: a randomised controlled trial comparing roboticassisted vs. standard laparoscopic surgery for rectal cancer resection. Trials. 2018;19:339.

12. Hahnloser D. The robot: a big disappointment, or just early days? Colorectal Dis. 2018;20:751-752.

13. Rahbari NN, Weitz J, Hohenberger W, et al. Definition and grading of anastomotic leakage following anterior resection of the rectum: a proposal by the International Study Group of Rectal Cancer. Surgery. 2010;147:339-351.

14. Munoz M, Acheson AG, Auerbach M, et al. International consensus statement on the peri-operative management of anaemia and iron deficiency. Anaesthesia. 2017;72:233-247.

15. Duchalais E, Machairas N, Kelley SR, et al. Does prolonged operative time impact postoperative morbidity in patients undergoing robotic-assisted rectal resection for cancer? Surg Endosc. 2018;32:3659-3666.

16. Merchea A, Ali SM, Kelley SR, et al. Long-term oncologic outcomes of minimally invasive proctectomy for rectal adenocarcinoma. Journal of Gastrointestinal Surgery. 2018;22:1412-1417.

17. Larson DW, Lovely JK, Cima RR, et al. Outcomes after implementation of a multimodal standard care pathway for laparoscopic colorectal surgery. BJS. 2014;101:1023-1030.

18. Prete FP, Pezzolla A, Prete F, et al. Robotic versus laparoscopic minimally invasive surgery for rectal cancer: a systematic review and meta-analysis of randomized controlled trials. Ann Surg. 2018;267:1034-1046.
19. Sun Y, Xu H, Li Z, et al. Robotic versus laparoscopic low anterior resection for rectal cancer: a meta-analysis. World J Surg Oncol. 2016;14:61.

20. Hirst A, Philippou Y, Blazeby J, et al. No surgical innovation without evaluation: evolution and further development of the IDEAL framework and recommendations. Ann Surg. 2018;269:211-220.

21. Hellan M, Ouellette J, Lagares-Garcia JA, et al. Robotic rectal cancer resection: a retrospective multicenter analysis. Ann Surg Oncol. 2015;22:2151-2158.

22. Baek SJ, Kim CH, Cho MS, et al. Robotic surgery for rectal cancer can overcome difficulties associated with pelvic anatomy. Surg Endosc. 2015;29:1419-1424.

23. Park JS, Kim NK, Kim SH, et al. Multicentre study of robotic intersphincteric resection for low rectal cancer. Br J Surg. 2015;102:1567-1573.

24. Valverde A, Goasguen N, Oberlin O, et al. Robotic versus laparoscopic rectal resection for sphincter-saving surgery: pathological and short-term outcomes in a single-center analysis of 130 consecutive patients. Surg Endosc. 2017;31:4085-4091.

25. Trastulli S, Farinella E, Cirocchi R, et al. Robotic resection compared with laparoscopic rectal resection for cancer: systematic review and meta-analysis of short-term outcome. Colorectal Dis. 2012;14:e134-e156.

26. Greer NL, Gunnar WP, Dahm P, et al. Enhanced recovery protocols for adults undergoing colorectal surgery: a systematic review and meta-analysis. Dis Colon Rectum. 2018;61:1108-1118.

27. Crippa J, Mari GM, Miranda A, et al. Surgical stress response and enhanced recovery after laparoscopic surgery - a systematic review. Chirurgia (Bucur). 2018;113:455-463.

28. Greco M, Capretti G, Beretta L, et al. Enhanced recovery program in colorectal surgery: a meta-analysis of randomized controlled trials. World $J$ Surg. 2014;38:1531-1541.

29. Pache B, Hubner M, Jurt J, et al. Minimally invasive surgery and enhanced recovery after surgery: the ideal combination? J Surg Oncol. 2017;116:613-616.

30. Jayne D, Pigazzi A, Marshall H, et al. Effect of robotic-assisted vs conventional laparoscopic surgery on risk of conversion to open laparotomy among patients undergoing resection for rectal cancer: the ROLARR randomized clinical trial. JAMA. 2017;318:1569-1580. 